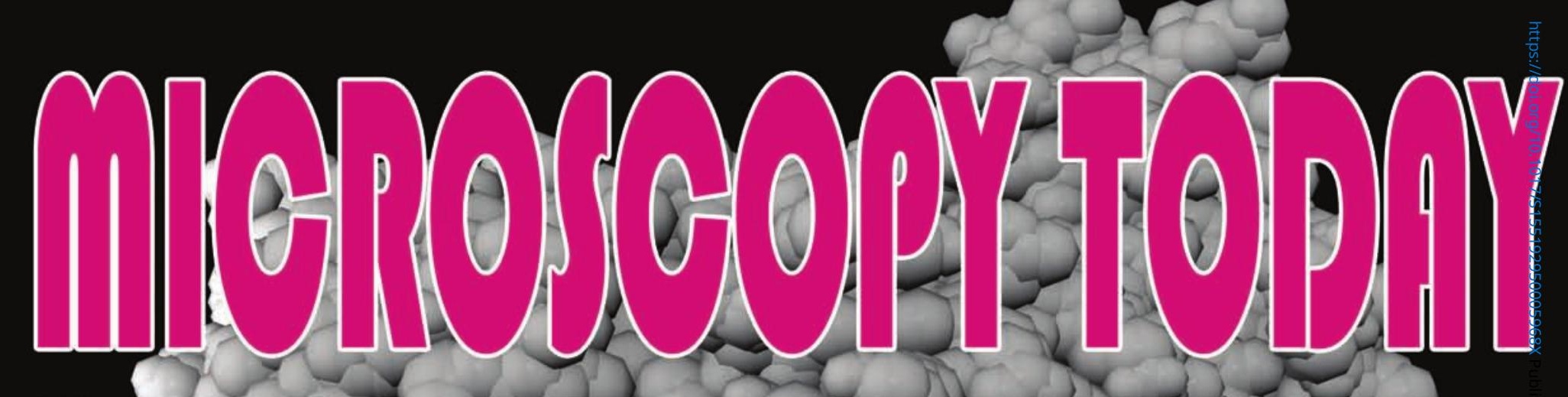

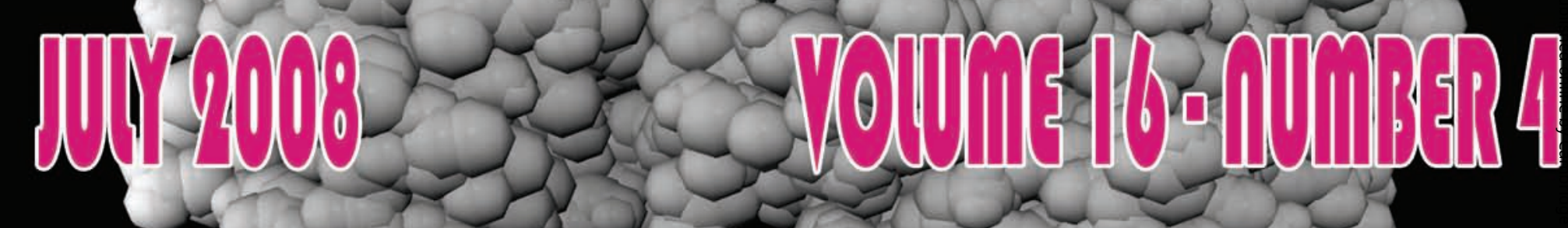

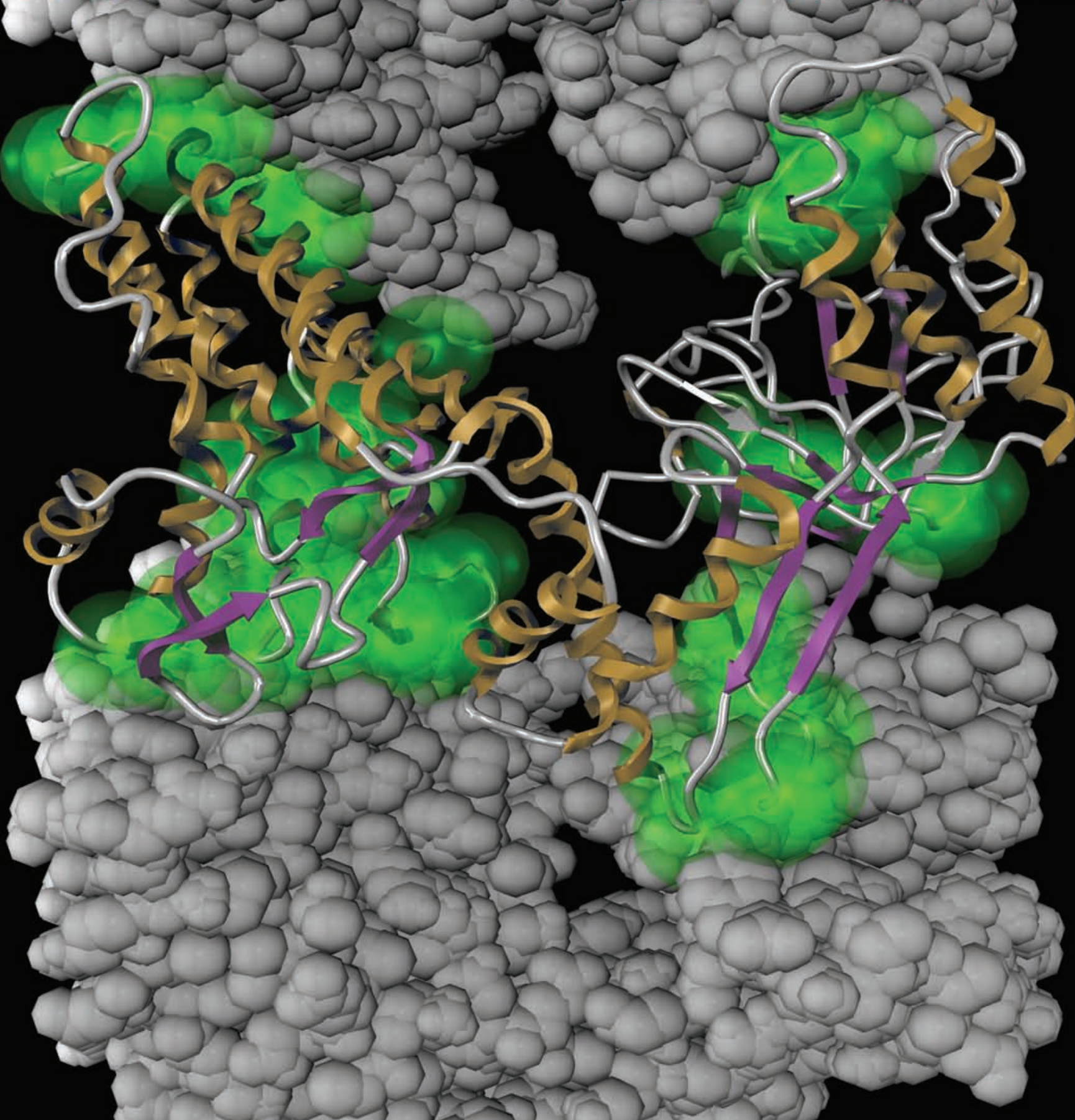




\section{Explore and Identify}

\section{Your Partner for Material Analysis}

\section{and Life Science}

Come and See us at

M\&M 2008,

Booth \# 804, Albuquerque, NM, August 3-7, 2008
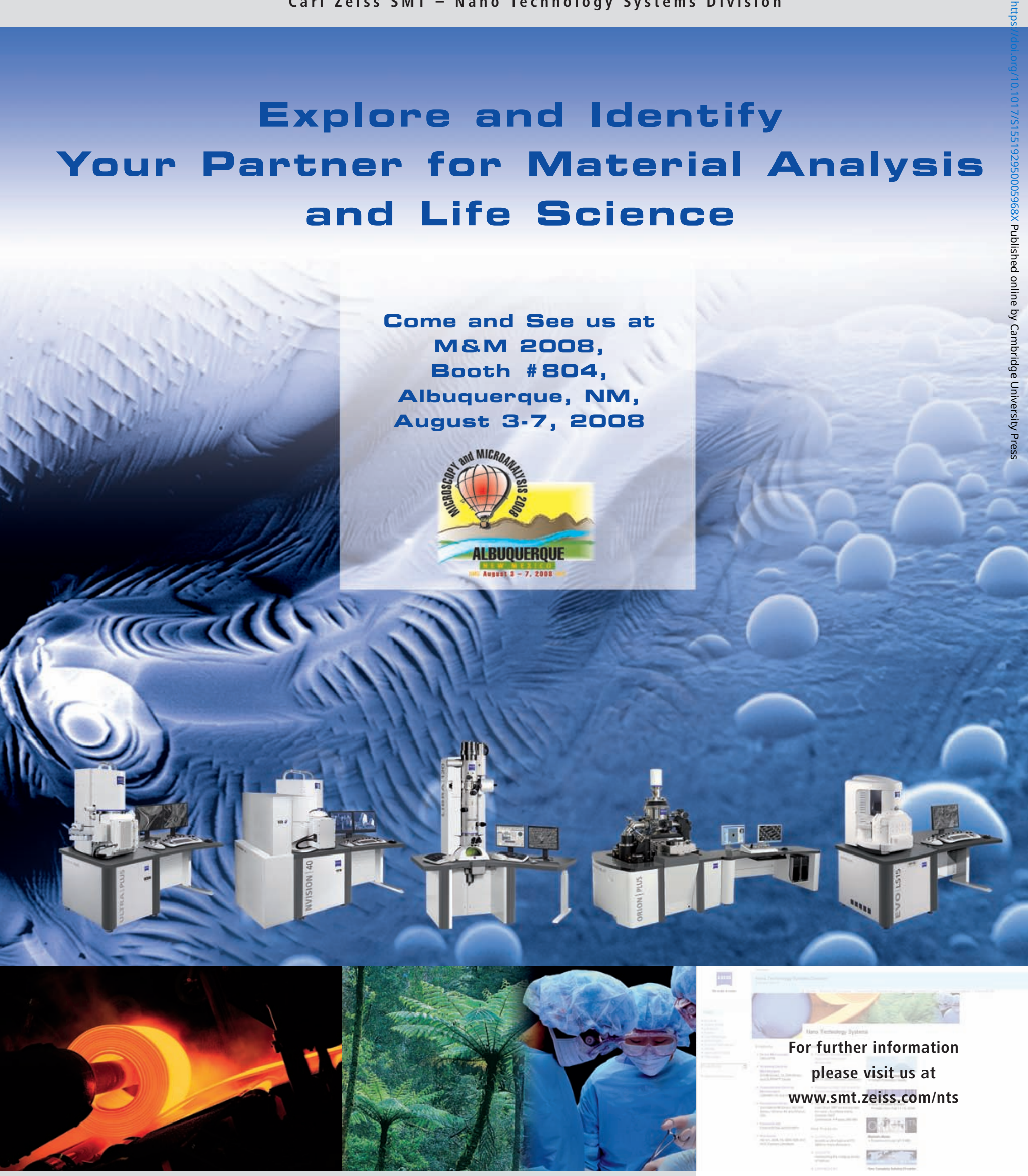


\section{How Much Force Does it Take to Move}

\section{an Atom on a Surface?}

\section{Stephen W. Carmichael ${ }^{1}$ Mayo Clinic}

It was demonstrated 18 years ago that atoms could be manipulated, one at a time, on a surface. Yet only recently has the force required to move an atom been determined. Markus Ternes, Christopher Lutz, Cyrus Hirjibehedin, Franz Giessibl, and Andreas Heinrich, in a technical tour de force, have engineered a microscope that incorporates features of the scanning tunneling microscope (STM) and atomic force microscope (AFM) to accurately quantitate the lateral and vertical forces needed to move a single atom on a surface. ${ }^{2}$

The STM was a breakthrough in the 1980's when it ushered in a new era of scanning probe microscopes. Of these new microscopes, the AFM is perhaps the most widely used these days. Each instrument has its advantages and limitations, but the best features of both have not previously been combined into a single instrument. At the "heart" of this new instrument is a small quartz tuning fork, with one fixed prong and the free end with a sharp tip. The free prong acts as a stiff cantilever in an AFM; variations in its longitudinal bending can be detected by measuring tiny piezoelectric currents that result when the quartz deforms. The stiff cantilever allows stable, small amplitude oscillation close to a surface. It was used in the frequency modulated mode with the oscillation amplitude constant. A force detected between the tip and surface produces a quantifiable variation in the resonant frequency of the cantilever. Under the conditions of these experiments, this variation approximates the force gradient between the tip and surface. To allow comparisons with STM manipulations, Ternes et al. also measured the tunneling conductance between the tip and the surface.

Ternes et al. derived the force to move an atom from the measurement of the vertical stiffness of the junction between the tip and sample. By computing this as a function of both vertical and lateral tip position, they could derive the force to move an atom in either direction. They found that moving a cobalt atom $(\mathrm{Co})$ on a special surface of platinum
[Pt(111)] requires a lateral force of 210 picoNewtons $(\mathrm{pN})$ and this force is independent of the vertical force. Interestingly, the lateral force varied substantially with the underlying surface as it required only $17 \mathrm{pN}$ to move a Co atom on a copper surface $[\mathrm{Cu}(111)]$.

Essentially, Ternes et al. measured the lateral force by pulling on a Co atom with the probe until it "hopped" from its initial binding site (a small depression in the surface) to the next binding site. This was reproducible and the force required was consistently in the same range $( \pm 5 \%)$. The vertical force was determined by integrating the lateral force along the vertical axis in a series of line scans. They interpreted the vertical force as the sum of a background force (including van der Waals and other long range forces) and a short range force between the bound atom and the surface. The lateral force was determined by integrating the vertical force again along the vertical axis and differentiating the resulting potential laterally. The vertical force exerted on the moving atom was much larger than the lateral force, and could be varied within a wide range whereas the lateral moving force stayed constant. This suggested that the lateral force is the key for manipulation of metal atoms on flat metal surfaces.

The lateral force needed to move a symmetrical (spherical) Co atom was an order of magnitude less than that required to move an asymmetrical molecule (carbon monoxide). The spatial dependence of the forces was also found to be markedly different.

The methods developed by Ternes et al. provide important information about the manipulation process of individual atoms and molecules based on direct observations. This opens the way for exploration of atomicscale friction and atom and molecule diffusion on surfaces. It will also lead to a deeper insight into controlled assembly mechanisms from the bottom up. The driving mechanism to create future nanoscale devices can now be explored in a quantitative manner.

1 The author gratefully acknowledges Dr. Markus Ternes for reviewing this article.

2 Ternes, M., C.P. Lutz, C.F. Hirjibehedin, F.J. Giessbl, and A.J. Heinrich, The force needed to move an atom on a surface, Science 319:1066-1069, 2008. See also Perspectives article by O. Custance and S. Morita, How to move an atom, Science 319:1051-1052, 2008

\section{InPEX Of ARtIOLES}

How Much Force Does it Take to Move an Atom on a Surface? 3 Stephen W. Carmichael, Mayo Clinic

Beyond the Black Box: Interactive Global Docking of Protein Complexes

Jochen Heyd, Stefan Birmanns, University of Texas at Houston, School of Health Information Sciences, Houston, Texas

Comparing Binary Image Analysis Measurements Euclidean Geometry, Centroids and Corners Dennis W. Hetzner, Timken, Co. Canton, Ohio

Confocal Laser Microscopy on Biofilms: Successes and Limitations

Betsey Pitts and Philip Stewart, Center for Biofilm Engineering, Montana State University, Bozeman, Montana

Extreme High-Resolution SEM: A Paradigm Shift 24 R. Young ${ }^{\star}$, T. Templeton $*$ L. Roussel ${ }^{* *}$, I. Gestmann ${ }^{* *}$, G. van Veen ${ }^{* *}$, T. Dingle ${ }^{* *}$ and S. Henstra ${ }^{* *}$ FEI, ${ }^{*}$ Hillsboro, OR and ${ }^{*}$ Eindhoven, The Netherlands

Testing Parameters for Two-Dimensional Crystallization and Electron Crystallography on Eukaryotic Membrane Proteins with Liposomes as Controls

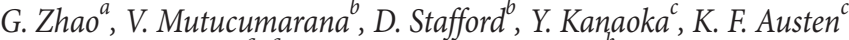
and I. Schmidt-Krey ${ }^{a},{ }^{a} G A$ Inst. of Tech., Atlanta, ${ }^{b}$ UNC, Chapel Hill, NC, ${ }^{c}$ Harvard and Brigham and Women's Boston, MA

A New Perspective on Mechanical Testing: In Situ Compression in the TEM

Julia Deneen Nowak, Zhiwei Shan and Oden L.

Warren, ${ }^{*}$ Hysitron Incorporated, Minneapolis, MN

Confocal Micro X-Ray Fluorescence: A New Paradigm

in Materials Characterization

Brian M. Patterson, George J. Havrilla, Kimberly A. DeFriend, LANL, Los Alamos, NM

\section{Hardware and Techniques for Cross-Correlative} TEM and Atom Probe Analysis

B.P. Gorman ${ }^{1}$ D. Diercks, ${ }^{1}$ N. Salmon, ${ }^{2}$ E. Stach, ${ }^{2}$ G. Amador, ${ }^{3}$ C. Hartfield, ${ }^{3}$ U. of North Texas, Denton, TX, ${ }^{2}$ Hummingbird Scientific Instruments, Salem, OR, ${ }^{3}$ Omniprobe, Inc., Dallas, TX

Imaging Skin Epidermal Stem Cells: A Review ............. 48 Hilda Amalia Pasolli, The Rockefeller University New York, NY

Signature Analysis Applied to EDS Microanalysis

J.W. Colby \& D. C. Ward, xk, Incorporated, Clackamas, OR

Cryo-Fracture or Freeze-Fracture, a Method to Expose Internal Tissue Surfaces and Cell Surfaces for Viewing in the Scanning Electron Microscope 56

Jeannette Taylor, Emory University, Atlanta, Georgia

SEM Remote Control with a 3D Option .60

F. Mighela, C. Perra, R. Pintus, S. Podda, M. Vanzi, Sardegna Ricerche, Pula, Italy and DIEE, U. of Cagliari, Cagliari, Italy

Coloring Pictures for Electron Microscopists or Elements of Digital Image Manipulation for Students

V.M.Dusevich, J.H.Purk, J.D.Eick, University of Missouri, Kansas City, $M O$

Industry News 64

NetNotes

Advertiser's Index

(1) 9BOUT THE COVER

The cover shows three subunits of the chaperonin GroEL, with favorable interactions highlighted by green spheres. All monomers were docked into an experimental $3 \mathrm{D}$ reconstruction of the chaperoning using Interactive Global Docking (see article on page 6). 